\title{
Rauchfreie Psychiatrie - eine Illusion
}

\author{
Psychiatry Without Smoking is an Illusion
}

Bibliografie

DOI $10.1055 / \mathrm{s}-2007-986217$

Psychiat Prax 2008; 35: 5-7

(c) Georg Thieme Verlag KG

Stuttgart · New York .

ISSN 0303-4259

Korrespondenzadressen

PD Dr. med. Katja Cattapan-

Ludewig

Universitätsklinik und Poliklinik für Psychiatrie, Universität Bern Murtenstraße 21

3010 Bern, Schweiz

katja.cattapan@puk.unibe.ch

\section{Prof. Dr. Anil Batra}

\section{Dr. Friederike D. Wernz}

Universitätsklinik für Psychiatrie und Psychotherapie Tübingen

Osianderstraße 24

72076 Tübingen

anil.batra@med.uni-

tuebingen.de

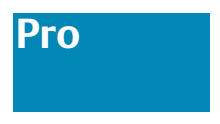

Es ist nicht zu leugnen: Rauchen hat schwerwiegende Folgeschäden und lange Zeit wurde Nikotinabhängigkeit (ICD-10 F17.2) von den Psychiatern zu wenig als Suchterkrankung wahrgenommen. Bei Patienten mit psychiatrischen Erkrankungen ist die Prävalenz des Rauchens im Vergleich zur psychiatrisch gesunden Allgemeinbevölkerung zwei- oder mehrfach erhöht; ungefähr 45-88\% der Schizophreniepatienten (bei Hospitalisierung: 82\%), 53-66\% der Patienten mit posttraumatischer Belastungsstörung und $40-60 \%$ der Depressionspatienten im Vergleich zu 23\% der Allgemeinbevölkerung sind Raucher; auch andere psychiatrische Erkrankungen gehen mit einer erhöhten Prävalenz des Rauchens einher (Daten aus den USA, zusammengefasst in [1]). Psychiatriepatienten sind oft sogenannte „heavy user“, d.h. die Anzahl an Zigaretten pro Tag und die Menge an inhalierter psychoaktiver Substanz pro Zigarette ist im Durchschnitt höher als bei vergleichbaren, psychiatrisch gesunden Kontrollpersonen, auch ist die Rückfallrate nach Entzug erhöht. Der Schweregrad der psychischen Störung ist assoziiert mit der Abhängigkeit von Nikotin. Schwere Nikotinabhängigkeit wird dabei als Ausdruck der individuellen psychopathologischen Vulnerabilität erachtet [2].

So sehr es zu wünschen wäre, dass all diese Patienten auf das Rauchen verzichten könnten, so halte ich dennoch die Forderungen für eine absolut rauchfreie Psychiatrie nicht für sinnvoll, werden dabei doch wichtige Aspekte des Zusammenhanges zwischen Nikotinkonsum und -entzug mit dem psychischen Befinden außer Acht gelassen. Folgende Argumente sprechen gegen ein generelles Rauchverbot in der Psychiatrie:

- Die erhöhte Prävalenz an Rauchern unter psychiatrisch Erkrankten und deren hohe Rückfallrate nach Nikotinentzug ist kein Zufall. Neben der mangelnden sozialen Stimulierung, die auch zum erhöhten Nikotinkonsum bei- tragen kann, gibt es biologische Faktoren, die für die stärkere Abhängigkeit, den besonders belastenden Nikotinentzug und die selten lang anhaltende Abstinenz bei Psychiatriepatienten relevant sind. Biologische Faktoren, die eine Nikotinabhängigkeit begünstigen, sind bei der Schizophrenie gut untersucht (zusammengefasst in [3]). Der cholinerge Nikotinrezeptor ist direkt involviert in die Pathophysiologie der Erkrankung. Nikotinkonsum kann als eine Art „kurz anhaltende Selbstmedikation“ verstanden werden, um Störungen von Informationsverarbeitung, Aufmerksamkeit und Kognition - also Basisdefizite dieser Erkrankung - zu verbessern. Nikotin hat auch einen neurobiologischen Bezug zu anderen psychiatrischen Erkrankungen, z. B. setzen Patienten mit Depression Nikotin zur Reduktion der Anhedonie ein [4].

- Versuche einer rauchfreien Psychiatrie zeigen, dass die Patienten nach der Entlassung aus der Institution nicht nikotinabstinent bleiben können. Psychiatrische Patienten, die in einer rauchfreien psychiatrischen Klinik in Kalifornien hospitalisiert waren, wurden nach Entlassung mittels Verlaufsuntersuchungen bezüglich ihrer Rauchgewohnheiten untersucht: Nach drei Monaten hatten alle der 90 untersuchten Patienten wieder begonnen zu rauchen, 76\% sogar schon am Tag nach der Entlassung, viele sogar schon innerhalb von fünf Minuten nach ihrem Austritt aus der Klinik [5]. Dies zeigt, wie wenig nützlich ein generelles Rauchverbot in einer psychiatrischen Klinik ist.

- Rauchen verändert die Plasmaclearance einiger Psychopharmaka über eine durch den Zigarettenrauch vermittelte Induktion der für den Abbau von Psychopharmaka relevanten Zytochrome $\mathrm{P}_{450}$ CYP $_{1 \mathrm{A2}}$ und $\mathrm{CYP}_{2 \mathrm{E} 1}$ und der Glukuronidierung (Pharmakokinetik). Außerdem kann die psychopharmakologische The- 
rapie aufgrund nikotinassoziierter pharmakodynamischer Prozesse verändert werden. Daher können Veränderungen der Rauchgewohnheiten einen Einfluss auf die Medikamentenwirkungen haben; der Einfluss des Rauchens wird insbesondere auf die Plasmakonzentrationen einiger Antipsychotika wie Clozapin und Olanzapin, aber auch in geringerem Ausmaß bei Antidepressiva wie Imipramin, Clomipramin, Fluvoxamin und Mirtazapin und verschiedenen Benzodiazepinen beschrieben (zusammengefasst in [6]). So kann ein plötzlicher Nikotinentzug potenziell gefährlich sein, wenn der Spiegel des Psychopharmakons deshalb in einen toxischen Bereich steigt [7]. Auch eine Veränderung in die andere Richtung, die Wiederaufnahme des Rauchens, kann Folgen haben. Ein Beispiel: Ein in einer rauchfreien psychiatrischen Klinik mit Clozapin eingestellter Schizophreniepatient beginnt nach der Entlassung zu Hause wieder zu rauchen wovon man aufgrund der extrem hohen Rückfallrate auszugehen hat [5] -, bei diesem kann es aufgrund der oben beschriebenen Interaktionen und der daraus resultierenden Abnahme des Plasmaspiegels an Clozapin zu einer deutlichen Verminderung der antipsychotischen Wirkung kommen. Deshalb wäre es für eine effiziente und nebenwirkungsarme Einstellung der Pharmakotherapie sinnvoll, wenn Patienten keine größeren Veränderungen der Rauchgewohnheiten während und nach dem Klinikaufenthalt hätten. Bei Veränderungen der Rauchgewohnheiten muss die Psychopharmakotherapie sorgfältig monitorisiert werden.

- Bis zu 30\% der Patienten kommen unfreiwillig in unsere psychiatrischen Kliniken. Diese Menschen befinden sich meist in einer existenziellen Krise, sie erleben bei ihrer Aufnahme oft bedrohliche psychotische Zustände oder quälende Suizidimpulse. Der Eintritt in die meist geschlossenen Abteilungen unserer Kliniken ist für diese Patienten ein großer Einschnitt in ihre persönliche Freiheit. Aufgabe der Klinik ist es, die Grunderkrankungen zu behandeln und Einschränkungen der Autonomie nur dann vorzunehmen, wenn sie relevant sind für eine effiziente Therapie oder wenn die Verhaltensweisen unzumutbar für die Umwelt sind. Es erscheint ethisch und juristisch sehr fragwürdig, ob die Persönlichkeitsrechte unter solchen ohnehin sehr schwierigen Umständen stärker beschnitten werden dürfen, als unbedingt nötig.

\section{Fazit}

Ein erzwungenes Rauchverbot hat keinen nachhaltigen Nutzen für psychiatrische Patienten, da die Rückfallrate sehr hoch ist. Erkenntnisse über die biologischen Zusammenhänge zwischen der Pathophysiologie psychiatrischer Erkrankungen und dem Konsum von Nikotin weisen zudem auf objektive und subjektive Effekte hin, die bei der individuellen Beurteilung der Vor- und Nachteile eines ohnehin nur vorübergehenden vollständigen Nikotinentzugs ebenfalls berücksichtigt werden müssen. Schließlich können Veränderungen des Nikotinkonsums zu gefährlichen Veränderungen der Serumspiegel von Psychopharmaka führen. Unter diesen Voraussetzungen würde es sich bei einem generellen Rauchverbot in der Psychiatrie um eine unzumutbare Einschränkung der Patientenautonomie handeln.

Diese Positionierung gegen ein generelles Rauchverbot schließt natürlich keineswegs aus, psychiatrische Patienten zu motivieren und zu unterstützen, ihren Nikotinkonsum zu reduzieren oder aufzugeben. In unserer eigenen Institution haben wir zudem sehr gute Erfahrungen mit Rauchfreiheit in allen öffentlichen Zonen gemacht, was von Nichtrauchern und Personal sehr geschätzt und selbst von den rauchenden Patienten praktisch ausnahmslos akzeptiert und respektiert wird. Raucherentwöhnungen sollten nicht mit der Behandlung einer akuten psychischen Erkrankung vermischt werden, sondern vor allem im ambulanten Rahmen oder in Rehabilitationseinrichtungen bei motivierten Patienten in Phasen psychischer Stabilität und aufgrund der pharmakologischen Interaktionen - unter ärztlicher Überwachung durchgeführt werden.

\section{Kontra}

Die Prävalenz des Rauchens ist bei Personen mit psychiatrischen Erkrankungen höher als in der psychisch gesunden Bevölkerung. Dies trifft insbesondere auf Patienten mit einer schizophrenen Psychose, mit einer Abhängigkeit von Alkohol oder illegalen Drogen sowie mit affektiven Störungen zu. Die Ursachen für die hohen Raucherprävalenzen, aber auch den überdurchschnittlich hohen Tageszigarettenkonsum und die geringen Abstinenzquoten bei Aufhörversuchen liegen dabei z.T. in störungsspezifischen Bedingungen: die antidepressive Wirkung des Nikotins und vermutlich auch weiterer Komponenten des Tabakrauchs, die synergistische Stimulation des „Belohnungszentrums“ (Ncl. accumbens) durch Nikotin und Alkohol bzw. illegale Drogen und die positiven Wirkungen des Rauchens auf die kognitiven Funktionen sowie manche neuroleptikainduzierten Nebenwirkungen bei Patienten mit einer Schizophrenie sind nur einige Beispiele für neurobiologische Befunde, die den Zusammenhang des Rauchens mit psychiatrischen Erkrankungen erklären helfen $[8,9]$. Daneben sind aber auch soziale Bedingungen und letztlich auch die permissive Umgebung für die hohen Konsumraten verantwortlich zu machen. So wird das Rauchen oft auch als ein Ausdruck der Lebensqualität angesehen [10]. Behandler in psychiatrischen Einrichtungen halten eine Intervention nicht selten für aussichtslos oder angesichts der akuten psychischen Problematik für nachrangig.

In den letzten Jahren hat ein Umdenken eingesetzt: die Belege für die tabakrauchbedingte gesundheitliche Gefährdung der psychiatrischen Patienten sind eindeutig [11] und unterstützen die Forderungen, auch dieser Zielgruppe Tabakentwöhnungen anzubieten [12]. Einige Studien konnten zeigen, dass die Bereitschaft zur Abstinenz groß ist und Entwöhnungsangebote durchaus erfolgreich sein können [13]. Im Ausland liegen bereits Erfahrungen mit der Einführung rauchfreier psychiatrischer Kliniken vor: Erste versuchsweise Rauchverbote in US-amerikanischen psychiatrischen Krankenhäusern werden in der Literatur positiv bewertet - das Rauchverbot unmittelbar nach stationärer Aufnahme führte wider Erwarten nicht zu einer signifikanten Verschlechterung der psychiatrischen Symptomatik, auch wenn ein Nikotinentzug auftrat [14]. Hempel et al. [15] beschreiben die Effekte eines Rauchverbotes, das 1998 in einer geschlossenen forensischen Einrichtung ausgesprochen wurde: Überraschenderweise tolerierten nicht nur Patienten (und Personal) das Rauchverbot, es kam in der Folge sogar zu einer Reduktion gewaltsamen Verhaltens in der Einrichtung. Mittlerweile liegen mehr als 26 Studien zum Effekt eines Rauchverbotes in psychiatrischen Kliniken auf die Psychopathologie, Behandlungscompliance und die langfristige Tabakabstinenz vor [16]. Die im Vorfeld geäußerten Befürchtungen des Personals hinsichtlich einer Eskalation von Gewalt und Unruhe traten nicht ein.

Im Zuge der allgemeinen Bestrebungen, einen wirksamen Gesundheitsschutz vor Tabakrauch zu schaffen, beschloss die deutsche Regierung, dem Beispiel anderer Länder folgend, ein Rauch- 
verbot in öffentlichen Einrichtungen, in Verkehrsmitteln, in der Gastronomie sowie in Schulen und in Krankenhäusern. Das Rauchverbot in Krankenhäusern könnte dabei nicht nur Patienten und Mitarbeiter vor der Tabakrauchexposition schützen, sondern zugleich die zahlreichen professionellen Multiplikatoren animieren, Rauchern Angebote zum Ausstieg zu vermitteln [17].

In Deutschland tun wir uns offenbar schwer mit der radikalen Umsetzung eines totalen Rauchverbotes: insbesondere mit Hinweis auf die Freiheit des Einzelnen wird für Patienten, die in einer akuten psychischen Krise - häufig gegen ihren Willen - in einer psychiatrischen Klinik untergebracht sind, eine Ausnahmeregelung gefordert. Dies hat Auswirkungen auf die Gestaltung der derzeit auf Länderebene verabschiedeten „Nichtraucherschutzgesetze“, die einerseits ein Rauchverbot in Krankenhäusern, andererseits Ausnahmen für psychiatrische Kliniken vorsehen. Im baden-württembergischen Landesnichtraucherschutzgesetz ist folgende weitreichende Ausnahme verankert ( $\$ 6$ Abs. 2): „Abweichend... können in Krankenhäusern Ausnahmen für solche Patientinnen und Patienten zugelassen werden, die... sich zu einer psychiatrischen Behandlung oder aufgrund einer gerichtlich angeordneten Unterbringung in einer geschlossenen Abteilung des Krankenhauses aufhalten oder bei denen die Untersagung des Rauchens dem Therapieziel entgegensteht. Die Entscheidung, ob im Einzelfall das Rauchen erlaubt werden soll, trifft die behandelnde Ärztin oder der behandelnde Arzt.“

Trotz mancher Vorbehalte wurde in Tübingen im Jahr 2001 der Versuch unternommen, die Universitätsklinik für Psychiatrie und Psychotherapie „rauchfrei“ zu machen. Geraucht wurde bis dahin in fast allen Bereichen der Klinik. Eine Arbeitsgruppe von rauchenden und nicht rauchenden Mitarbeitern der Verwaltung, des Pflegedienstes und des Ärztlichen Dienst sowie Personalratsangehörigen initiierte einen demokratischen Prozess zur Änderung der „Rauchkultur“ in der Klinik. Über 300 Mitarbeiter des Hauses wurden zur Notwendigkeit und Akzeptanz von Rauchregulationen in der Klinik befragt. Fast alle Nichtraucher (94,7\%) und die Mehrheit der Raucher (73,5\%) sprachen sich für Rauchregulationen und die Einrichtung eines Raucherbereiches im Garten der Klinik aus. Daraufhin wurde ein generelles Rauchverbot im Gebäude verfügt - Ausnahmen blieben die Raucherzimmer auf den beschützenden Stationen. In die Betriebsvereinbarung wurden dafür Therapieangebote zur Tabakentwöhnung für Mitarbeiter und Patienten aufgenommen. Rauchende Patienten erhalten auf Wunsch eine temporäre Nikotinsubstitution und können nach Abklingen der akuten Symptomatik und noch während ihres stationären Aufenthaltes kostenfrei an einem Tabakentwöhnungskurs teilnehmen. Den Mitarbeitern stehen Angebote zur Teilnahme an Tabakentwöhnungskursen während ihrer Dienstzeit offen. Die medikamentöse Unterstützung wird ebenfalls subventioniert. Die Dienstvereinbarung wurde sowohl von Rauchern $(58 \%)$ als auch Nichtrauchern $(82,2 \%)$ positiv bewertetet.

Nach erfolgreicher Umsetzung der „Rauchfreien Psychiatrie“ (mit wenigen ausgewiesenen Raucherbereichen als Ausnahmeregelung und Angeboten zur Beratung und therapeutischen Unterstützung) wurde der Klinik im Jahr 2005 das Silberzertifikat des „Deutschen Netzes Rauchfreier Krankenhäuser“ verliehen.
Faktoren, die den Erfolg der Maßnahme bestimmt haben könnten, könnten die Einbeziehung aller Berufsgruppen, die Transparenz bezüglich der einzelnen Umsetzungsschritte und die Verbindung des Rauchverbotes mit therapeutischen Angeboten für Mitarbeiter und Patienten sein.

Obgleich das Rauchen bei Patienten mit psychischen Störungen u.a. auch die Funktion einer „Selbstmedikation“ übernimmt, scheint ein weitgehendes Rauchverbot in einer psychiatrischen Klinik realisierbar. Internationale Erfahrungen (und der eigene Versuch, eine vertretbare Lösung - eine „rauchfreie Klinik“ mit möglichst wenig definierten Ausnahmen - zu finden), zeigen deutlich, dass heute möglich ist, was lange als unvorstellbar galt. Wichtig für eine erfolgreiche Umsetzung des Rauchverbotes in einer Klinik ist der Grundsatz: Ein Rauchverbot dient dem Nichtraucherschutz, nicht der Benachteiligung der Raucher. Daneben besteht die Hoffnung, die Selbstverständlichkeit des Rauchens infrage stellen und ein klares Signal bezüglich der Unvereinbarkeit des Rauchens mit einer gesunden Lebensführung setzen zu können.

\section{Literatur}

1 Kalman D, Morissette SB, George TP. Co-morbidity of smoking in patients with psychiatric and substance use disorders. Am J Addict 2005; 14 (2): $106-123$

2 Martinez-Ortega JM, Jurado D, Martinez-Gonzalez MA, Gurpegui M. Nicotine dependence, use of illegal drugs and psychiatric morbidity. Addict Behav 2006; 31 (9): 1722 - 1729

3 Cattapan-Ludewig K, Ludewig S, Jaquenoud SE, Etzensberger M, Hasler F. Warum rauchen Schizophreniepatienten? Nervenarzt 2005; 76 (3): $287-294$

4 Laje RP, Berman JA, Glassman AH. Depression and nicotine: preclinical and clinical evidence for common mechanisms. Curr Psychiatry Rep 2001; 3 (6): 470-474

5 Prochaska JJ, Fletcher L, Hall SE, Hall SM. Return to smoking following a smoke-free psychiatric hospitalization. Am J Addict 2006; 15 (1): 15 22

6 Desai HD, Seabolt J, Jann MW. Smoking in patients receiving psychotropic medications: a pharmacokinetic perspective. CNS Drugs 2001; 15 (6): $469-494$

7 Haslemo T, Eikeseth PH, Tanum L, Molden E, Refsum H. The effect of variable cigarette consumption on the interaction with clozapine and olanzapine. Eur J Clin Pharmacol 2006; 62 (12): 1049-1053

8 Batra A. Tabakabhängigkeit und Raucherentwöhnung bei psychiatrischen Patienten. Fortschr Neurol Psychiatr 2000; 68: 80-92

9 Heinz A, Batra A. Neurobiologie der Alkohol- und Nikotinabhängigkeit. Stuttgart: Kohlhammer, 2003

10 Thielscher S. Tabakentwöhnung: Verwunderung. Dt Aerztebl 2007; 104: A-2258

11 Osborn DPJ. The poor physical health of people with mental illness. West J Med 2001; 175: 329-332

12 Hughes JR. Possible effects of smoke-free inpatient units on psychiatric diagnosis and treatment. J Clin Psychiatry 1993; 54 (3): 109-114

13 McNeill A. Smoking and patients with mental problems. Health Development Agency, 2004

14 Smith CM, Pristach CA, Cartagena M. Obligatory cessation of smoking by psychiatric inpatients. Psychiatr Serv 1999; 50: 91 - 94

15 Hempel AG, Kownacki R, Malin DH, Ozone SJ, Cormack TS, Sandoval BG, Leinbach $A E$. Effect of a total smoking ban in a maximum security psychiatric hospital. Behav Sci Law 2002; 20: 507-522

16 Lawn S, Pols R. Smoking bans in psychiatric inpatient settings? A review of the research. Aust N Z J Psychiatry 2005; 39: 866 - 885

17 Rustler C, Batra A. Rauchfreie Krankenhäuser: Mehr als nur Verbotszonen. Dt Aerztebl 2007; 104: A-1644 